\title{
Article
}

\section{Solvent Extraction of Various Metals Including Actinides by Bidentate and Tridentate Diamides}

\author{
Yuji Sasaki*, Takaumi Kimura \\ Japan Atomic Energy Agency, Tokai-mura, Ibaraki 319-1195, Japan \\ Koichi Oguma \\ Chiba University, Yayoi-cho, Inage-ku, Chiba 263-8522, Japan
}

(Manuscript submitted March 31, 2007; accepted May 9, 2007)

\begin{abstract}
Many diamide-type extractants were synthesized and tested for the extraction of actinides (III), (IV), (V) and (VI). The diglycolamides (DGA) with two amidic oxygen and one ether oxygen, which show the tridentate feature, have the high extractability of actinides (An). In order to increase the lipophilic property, the diglycolamides with the different functional groups attached to $\mathrm{N}$ atoms were synthesized and examined for their extractability. It was found that $N, N, N^{\prime}, N^{\prime}$-tetraoctyl-diglycolamide (TODGA) and $N, N, N^{\prime}, N^{\prime}$-tetradecyldiglycolamide (TDDGA) exhibit the satisfactory dissolution into $n$-dodecane with less solubility in $\mathrm{H}_{2} \mathrm{O}$. The results of various metal extractions using TODGA revealed that the divalent, trivalent and tetravalent metals with ionic radius of $90-120 \mathrm{pm}$ gave notably high $D$ values. After elucidation of the effect on substitution of the functional group, the chemical form of DGA to have high performance on An extraction was proposed.
\end{abstract}

Key words: DGA, actinide, lanthanide, solvent, extraction, diamide, tridentate

\section{Introduction}

The development of the novel extractants for the actinide (An) ions have been widely studied for the chemical treatment of the high level radioactive liquid waste (HLW). The diamide extractants can extract directly the metal ions from $\mathrm{HLW}$ of $3 \mathrm{M}\left(=\mathrm{mol} / \mathrm{dm}^{3}\right) \mathrm{HNO}_{3}$, because an ion-pair extraction using the neutral donor gives the high distribution ratio of metals from acid. One of the indispensable advantages of the diamide extractant is incinerability, after use diamide can be gasificated by combustion and produces no radioactive solid waste.

Many diamide extractants, i.e., malonamides, succinamide, glutalamide and adipamide, have been studied for the application to the recovery of An for the last two decades [1-5]. It is important to elucidate the effect on the structure of the diamide in order to investigate the best extractant for An. However, few papers have discussed An extraction abilities of the diamides with the different structural backbones [3].

In the present investigation, the structure of the central frame of the diamides with and without one ether oxygen or sulfur has been systematically changed and its influence to the extractability toward An(III), (IV), (V) and (VI) were studied. The diamides used here are: oxalamide $\left(\left(\mathrm{CONR}_{1}, \mathrm{R}_{2}\right)_{2}\right.$, abbreviated as OXA); malonamide $\left(\mathrm{CH}_{2}-\left(\mathrm{CONR}_{1}, \mathrm{R}_{2}\right)_{2}, \mathrm{MA}\right)$; succinamide $\left(\left(\mathrm{CH}_{2}\right)_{2}-\left(\mathrm{CONR}_{1}, \mathrm{R}_{2}\right)_{2}, \mathrm{SCA}\right)$; maleamide $\left((\mathrm{CH})_{2}-\left(\mathrm{CONR} \mathrm{N}_{1}, \mathrm{R}_{2}\right)_{2}\right.$, MLA); glutalamide $\left(\left(\mathrm{CH}_{2}\right)_{3}-\left(\mathrm{CONR}{ }_{1}, \mathrm{R}_{2}\right)_{2}\right.$, GLA); diglycolamide $\left(\mathrm{O}-\left(\mathrm{CH}_{2}-\mathrm{CONR}_{1}, \mathrm{R}_{2}\right)_{2}\right.$, DGA); oxydipropionamide (O$\left(\left(\mathrm{CH}_{2}\right)_{2}-\mathrm{CONR}{ }_{1}, \mathrm{R}_{2}\right)_{2}$, ODPA); thiadiglycolamide $\left(\mathrm{S}-\left(\mathrm{CH}_{2}-\mathrm{CONR}_{1}, \mathrm{R}_{2}\right)_{2}\right.$, TDGA); and thiadipropionamide (S$\left(\left(\mathrm{CH}_{2}\right)_{2}-\mathrm{CONR}_{1}, \mathrm{R}_{2}\right)_{2}$, TDPA). The structures of the examined diamides are illustrated in Fig. 1. Using these extractants, we have studied the effect of the structure of diamides on the solubility into the aqueous and organic diluents and metals extraction.

\subsection{Chemicals}

\section{Experimental}

The reagents used for the organic synthesis were commercially available. The standard solutions of lanthanides and the other non-radioactive metal ions for atomic absorption spectrometry (Wako Pure Chemical Industries, Ltd) were employed. The radioactive tracers of ${ }^{99} \mathrm{Tc},{ }^{233} \mathrm{U},{ }^{230} \mathrm{Th},{ }^{237} \mathrm{~Np}$ and ${ }^{241} \mathrm{Am}$ were obtained from Isotope Products Laboratories. All the other reagents were of analytical reagent grade.

\subsection{Organic Synthesis}


OXA was synthesized from the oxalic chloride and the secondary amines [6]. The initial reagents were mixed slowly in a chloroform media with the triethylamine, under the freezing point of water and stirred overnight. The product was then washed with successive, distilled water, $1 M \mathrm{HCl}$ and $1 M \mathrm{NaOH}$, and the materials obtained were purified by distillation and silica gel column chromatography. MA was synthesized from the malonic ester and the secondary amines [6]. The starting materials were mixed with hydrochloric acid and stirred at $200{ }^{\circ} \mathrm{C}$ for $1 \mathrm{~h}$. The product was dissolved in toluene and washed with successive, distilled water, $1 M \mathrm{HCl}$ and $1 M \mathrm{NaOH}$, and purified by distillation and silica gel column chromatography. Other diamides, such as SCA, MLA, GLA, DGA, TDGA, ODPA, and TDPA, were synthesized and purified by the same method as the ref. [7]. All diamides synthesized were identified by NMR (UNITY plus 400, Varian) and GC-MS (TurboMass, Perkin Elmer Inc.) spectrometers.

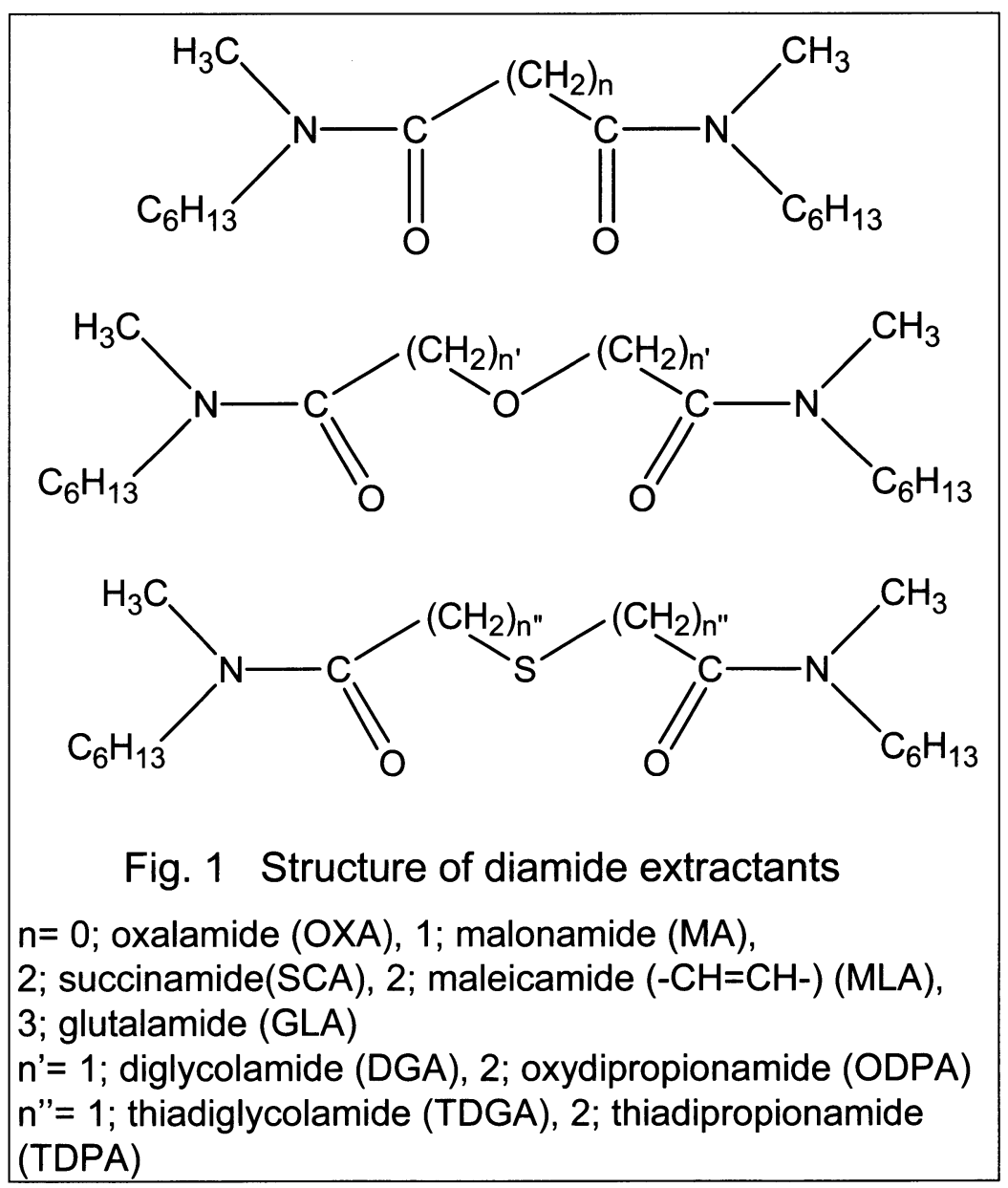

\subsection{Extraction Procedure}

Three $\mathrm{cm}^{3}$ of the organic solvent containing diamide of the concentration ranging from $5 \times 10^{-6}$ to $0.2 \mathrm{M}$ was shaken with an equal volume of $0.1 \mathrm{M}$ $\mathrm{NaClO}_{4}$ with $\mathrm{pH}=3$ or the nitric acid of the desired concentration. After centrifugation, $2 \mathrm{~cm}^{3}$ of the pre-equilibrated organic phase was taken and mixed with $2 \mathrm{~cm}^{3}$ of the aqueous phase of the same concentration as in pre-equilibration, spiked with $10^{-2} \mathrm{~cm}^{3}$ of the radioactive tracer solution or the non-radioactive metal solution. The mixture was shaken mechanically for $2 \mathrm{~h}$ at $25 \pm 0.1{ }^{\circ} \mathrm{C}$. After separation of the phases by centrifugation, duplicate $0.50 \mathrm{~cm}^{3}$ aliquots were measured by an $\mathrm{NaI}$ scintillation counter (COBRA 5003, Packard Instrument Company) for the gamma activities of ${ }^{241} \mathrm{Am}$, a liquid scintillation counter (Tri-Carb 1600 TR, Packard Instrument Company) for the alpha and beta activities of ${ }^{99} \mathrm{Tc}{ }^{230} \mathrm{Th},{ }^{233} \mathrm{U}$ and ${ }^{237} \mathrm{~Np}$ in 5 $\mathrm{cm}^{3}$ of ULTIMA-FLO (Packard Instrument Company) cocktail. The preparation and counting of ${ }^{237} \mathrm{~Np}(\mathrm{~V})$ were done in the manner described in reference [8]. The amounts of the non-radioactive metal ions in the sample solutions prepared from the aqueous and the organic phases were measured by an ICP-AES (SPS 3100, Seiko Instruments Inc)= or ICP-MS (SPQ 9000, Seiko-EG\&G).

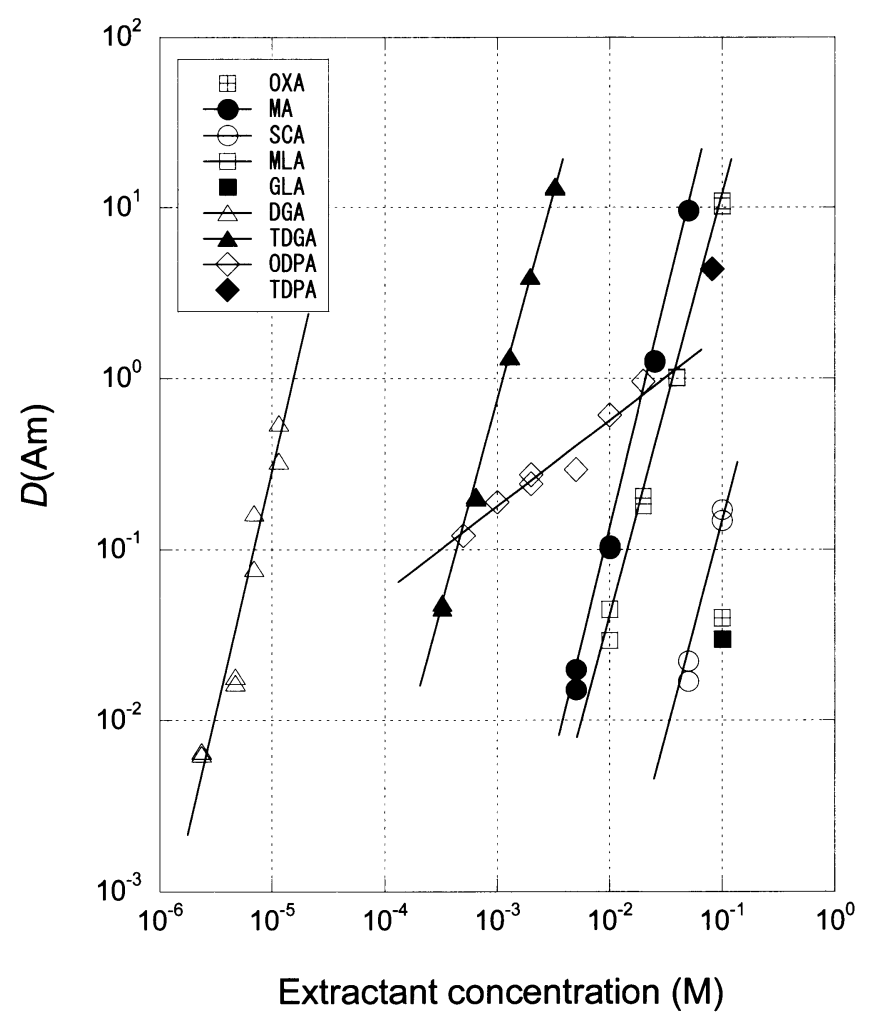

Fig. 2 Extraction of Am(III) by diamides

Organic diluent: nitrobenzene

Aqueous phase: $0.1 \mathrm{M} \mathrm{NaClO}_{4}, \mathrm{pH} 3.0$ 


\section{Results and Discussion}

\subsection{Extraction of Actinides by Different Diamides}

The distribution ratio, $D(\mathrm{Am})$, expressed by the ratio of $\mathrm{Am}$ (III) concentration in the organic phase to that in the aqueous phase, were shown in Fig. 2. The order of extractability of diamides for Am(III) is DGA $>$ TDGA > MA, ODPA > MLA, TDPA > SCA > GLA > OXA. $D(A m)$ by DGA is much higher than any other diamides in this work. TDGA, an analogue to DGA but with an ether sulphur atom, exhibits the second highest $D(\mathrm{Am})$. Assuming that $\mathrm{S}$ atom in TDGA coordinates hardly with the metal, TDGA would form an eight membered ring with metal ion similarly to GLA. TDGA showed much higher extractability toward Am(III) than that of GLA. This result strongly suggests the tridentate coordination by TDGA with three donors of two amidic oxygen and one ether sulfur. Slopes for the $\log D(\mathrm{Am})$ vs. $\log [$ extractant] in Fig. 2 are: $\operatorname{MA}(2.72 \pm 0.05)$; $\operatorname{SCA}(3.0 \pm 0.2) ; \quad \operatorname{MLA}(2.5 \pm 0.4) ; \operatorname{DGA}(2.73 \pm 0.09)$;

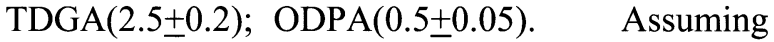
that the activity coefficient of the respective diamide is almost constant, these slope values are related to the number of the diamide molecules in the extraction reactions.

The distribution ratios of Th(IV), $D(\mathrm{Th})$, by the various diamides were plotted as a function of diamide concentration. The experimental condition used is identical with that of $\mathrm{Am}$ (III) extraction. The order of the extractability of diamides for Th(IV) is; DGA $>>$ TDGA $>$ MA, ODPA > MLA > SCA, GLA > TDPA > OXA. DGA also exhibits the highest $D$ values for Th(IV) extraction. The slopes of the plots in Fig. 3 were $1.5 \pm 0.2$ for MA, $1.7 \pm 0.3$ for SCA, $1.5 \pm 0.2$ for MLA, $0.97 \pm 0.08$ for DGA, $1.3 \pm 0.1$ for TDGA, $0.56 \pm 0.12$ for ODPA and 1.3 for TDPA. From the results of the slope analysis, the numbers of the diamide in the main extraction reactions are one or two.

In the previous study, a high $D(\mathrm{~Np})$ value has been obtained for the extraction of $\mathrm{Np}(\mathrm{V})$, ca. 10 by $0.06 \mathrm{M}$ DGA [8]. In the present study, $D(\mathrm{~Np})$ was measured using OXA, MA, SCA, MLA, GLA, TDGA, ODPA and TDPA. $N p(V)$ in the organic phase were under detection limit, thus $\mathrm{Np}(\mathrm{V})$ is poorly extracted by these extractants.

The distribution ratios of $\mathrm{U}(\mathrm{VI}), D(\mathrm{U})$, by the various diamides are shown in Fig. 4. The order of the extractability for U(VI) is DGA $>>$ ODPA > MA, TDGA > SCA, MLA > GLA > TDPA > OXA.

Both DGA and ODPA, introducing an ether $O$ atom into the central carbon chain, show higher

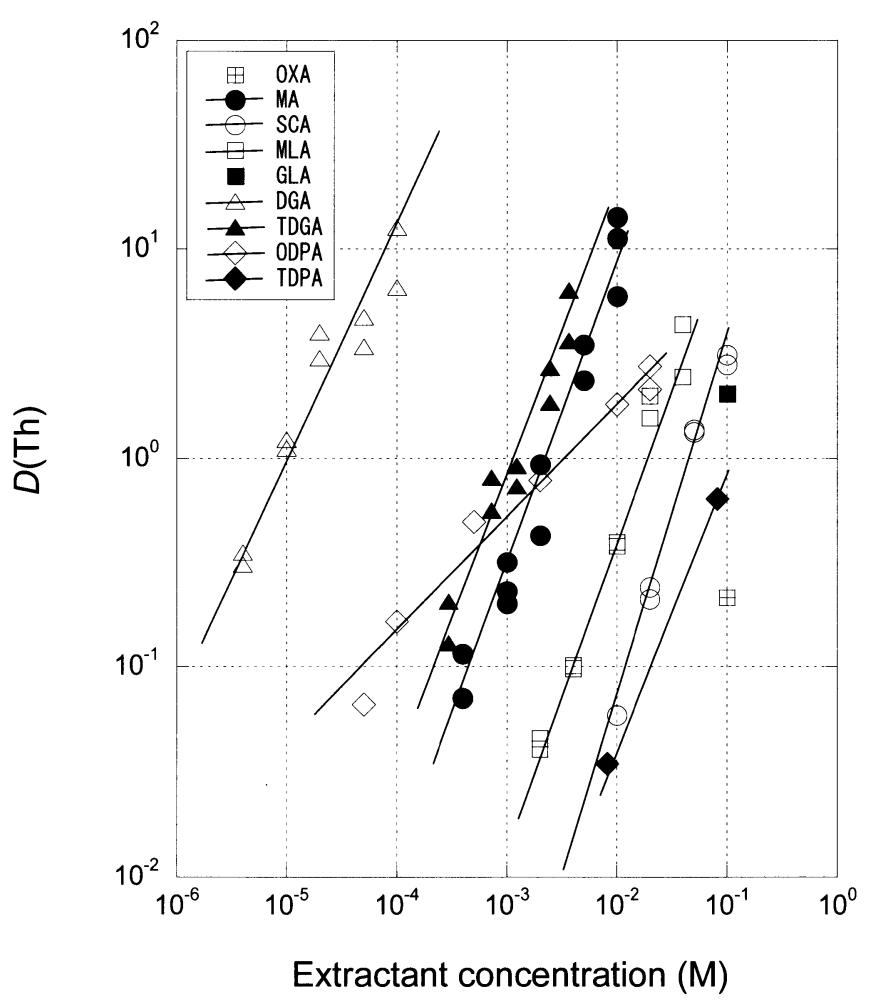

Fig. 3 Extraction of Th(IV) by diamides

Organic diluent: nitrobenzene

Aqueous phase: $0.1 \mathrm{M} \mathrm{NaClO}_{4}, \mathrm{pH} 3.0$

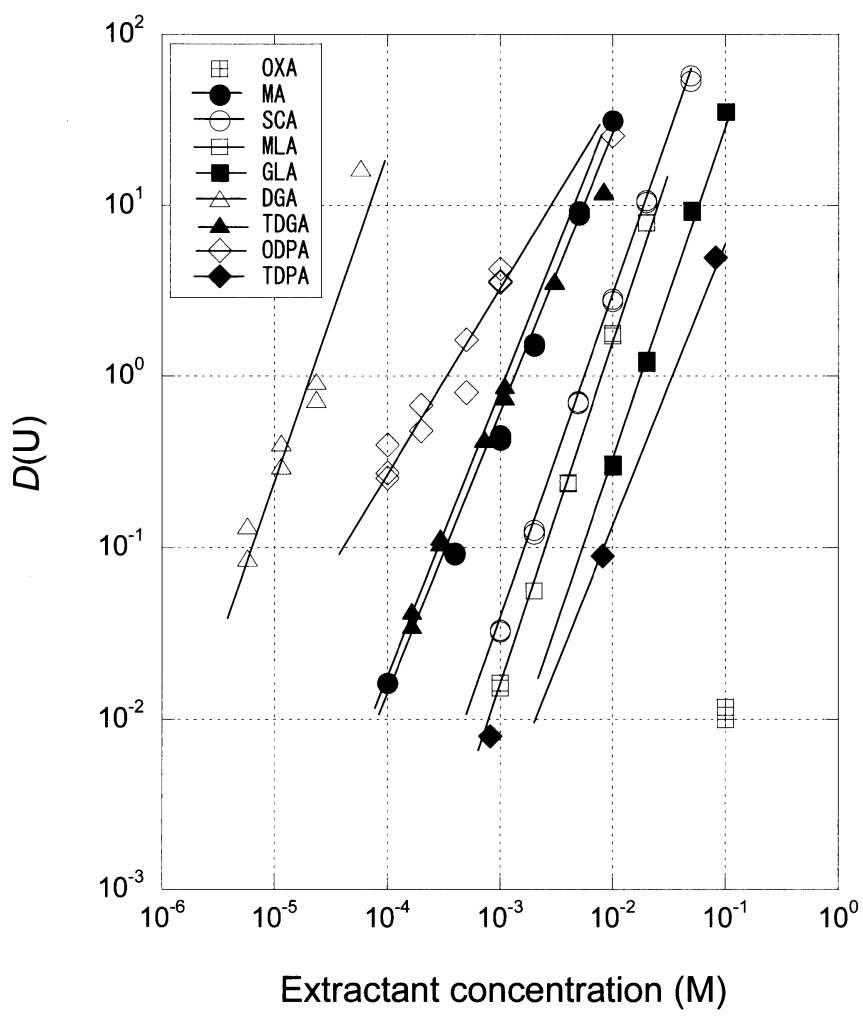

Fig. 4 Extraction of $\mathrm{U}(\mathrm{VI})$ by diamides

Organic diluent: nitrobenzene

Aqueous phase: $0.1 \mathrm{M} \mathrm{NaClO}_{4}, \mathrm{pH} 3.0$ 
Table 1 Solubility of DGA* in water and n-dodecane, and D of Am(III)

\begin{tabular}{lcccccc}
\hline & $n$ & $\begin{array}{c}\text { solubilily } \\
\text { in water }\end{array}$ & dissolution & \multicolumn{2}{c}{$D(\text { Am })^{* *}$} \\
\cline { 5 - 6 } & & in n-dodecane & chloroform & n-dodecane \\
\hline TPDGA & 3 & $57(\mathrm{mM})$ & poor & 0.41 & - \\
TBDGA & 4 & 2.3 & poor & 0.24 & - \\
TADGA & 5 & 0.25 & poor & 0.11 & - \\
THDGA & 6 & 0.11 & soluble & 0.089 & 40 \\
TODGA & 8 & 0.042 & unlimited & 0.071 & 30 \\
TDDGA & 10 & 0.042 & unlimited & 0.042 & 18 \\
\hline
\end{tabular}

*: General structure of DGA; O- $\left(\mathrm{CH}_{2} \mathrm{CON}\left(\mathrm{C}_{n} \mathrm{H}_{2 n+1}\right)_{2}\right)_{2}(\mathrm{n}=3,4,5,6,8,10)$

**: Determined by using 0.1M DGA/solvent and $1 \mathrm{M} \mathrm{HNO}_{3}$.

$D(\mathrm{U})$ than those by the other diamides. For SCA and MLA, forming the seven membered ring by two carbonyl oxygens, the $D(\mathrm{Am}, \mathrm{Th})$ with MLA is higher than those for SCA, whereas $D(\mathrm{U})$ with SCA is a little higher than that for MLA. As MLA has an ethylene bond in its backbone, MLA-metal complex is stabilized by the electronic conjugation, which may be more effective in Am and Th extractions. Whereas U(VI) has 4-6 coordination number in equatorial sites, MLA having $\mathrm{C}=\mathrm{C}$ double bond in the central frame may exhibit some restriction in $\mathrm{U}(\mathrm{VI})$-chelation. The slopes of the plots in Fig. 4 were calculated to be $1.7 \pm 0.2$ for MA, $1.9 \pm 0.2$ for SCA, $2.1 \pm 0.3$ for MLA, $2.1 \pm 0.4$ for GLA, $2.0 \pm 0.1$ for DGA, $1.5 \pm 0.1$ for TDGA, $1.0 \pm 0.1$ for ODPA and $1.4 \pm 0.2$ for TDPA, suggesting the number of the diamide in the main extraction reactions to be one or two.

\subsection{Modification to DGA Compound with High Lipophilic Property}

As an aliphatic diluent has been usually employed in the liquid-liquid extraction process involving HLW, the high solubility of the extractant into $n$-dodecane, is required. DGA compounds, which showed the highest extractability of An, with the different length of the alkyl chain attached to amidic $\mathrm{N}$ atoms were synthesized to see their physical properties. The existence of the long alkyl chain in an extractant may decrease the polarity of its metal complex and increase the solubility in the non-polar solvents. Six DGA compounds used here were O$\left(\mathrm{CH}_{2} \mathrm{CON}\left(\mathrm{C}_{\mathrm{n}} \mathrm{H}_{2 \mathrm{n}+1}\right)_{2}\right)_{2}$ with $\mathrm{n}=3$ (abbreviated as TPDGA), 4 (TBDGA), 5 (TADGA), 6 (THDGA), 8 (TODGA) and 10 (TDDGA). The solubility in water and $n$-dodecane, and $D(\mathrm{Am})$ using these samples were determined and the results were summarized in Table 1. TPDGA, TBDGA, TADGA and THDGA are slightly soluble in $n$ dodecane, and their $n$-dodecane solutions were turbid. The reason of the low solubility in $n$-dodecane is due to a high hydrophilicity of the DGA compounds. The carbon/oxygen $(\mathrm{C} / \mathrm{O})$ atom ratios in the molecules of TPDGA, TBDGA, TADGA and THDGA are 5.3, 6.7, 8 and 9.3, respectively. As these values, lower than those of the representative extractants for HLW treatment, i.e. O $\phi \mathrm{D}(\mathrm{iB}) \mathrm{CMPO} ; 12$ and DMDBTDMA; 13.5, signify their poor lipophilicity, TPDGA, TBDGA, TADGA and THDGA are not applicable to $\mathrm{HNO}_{3}$ - $n$-dodecane extraction system. The solubilities of six diglycolamides in water are shown in Table 1 . These values also support the fact that diglycolamides with shorter alkyl chain exhibit higher hydrophilic property. On the other hand, TODGA and TDDGA, which show the low solubility in water, can be dissolved in $n$-dodecane satisfactorily. Gasparini and Grossi suggested [9] that amide with more than 12-13 carbon atoms does not form a third phase. TODGA and TDDGA having 36 and 44 carbon atoms but with 3 oxygen ( $\mathrm{C} / \mathrm{O}$ ratio equal to 12 and 14.7) also indicated no third phase formation, even equilibrated with an aqueous solution of $6 M \mathrm{HNO}_{3}$. Distribution ratio of Am(III) in the system of $1 M \mathrm{HNO}_{3}$ and $0.1 M$ diglycolamide/ diluent are shown in Table 1, and it appears that the $D_{\mathrm{M}}$ values gradually decreased from TPDGA $(n=3)$ to TDDGA $(n=10)$, showing that longer alkyl chain gives some unstable complexing form. 

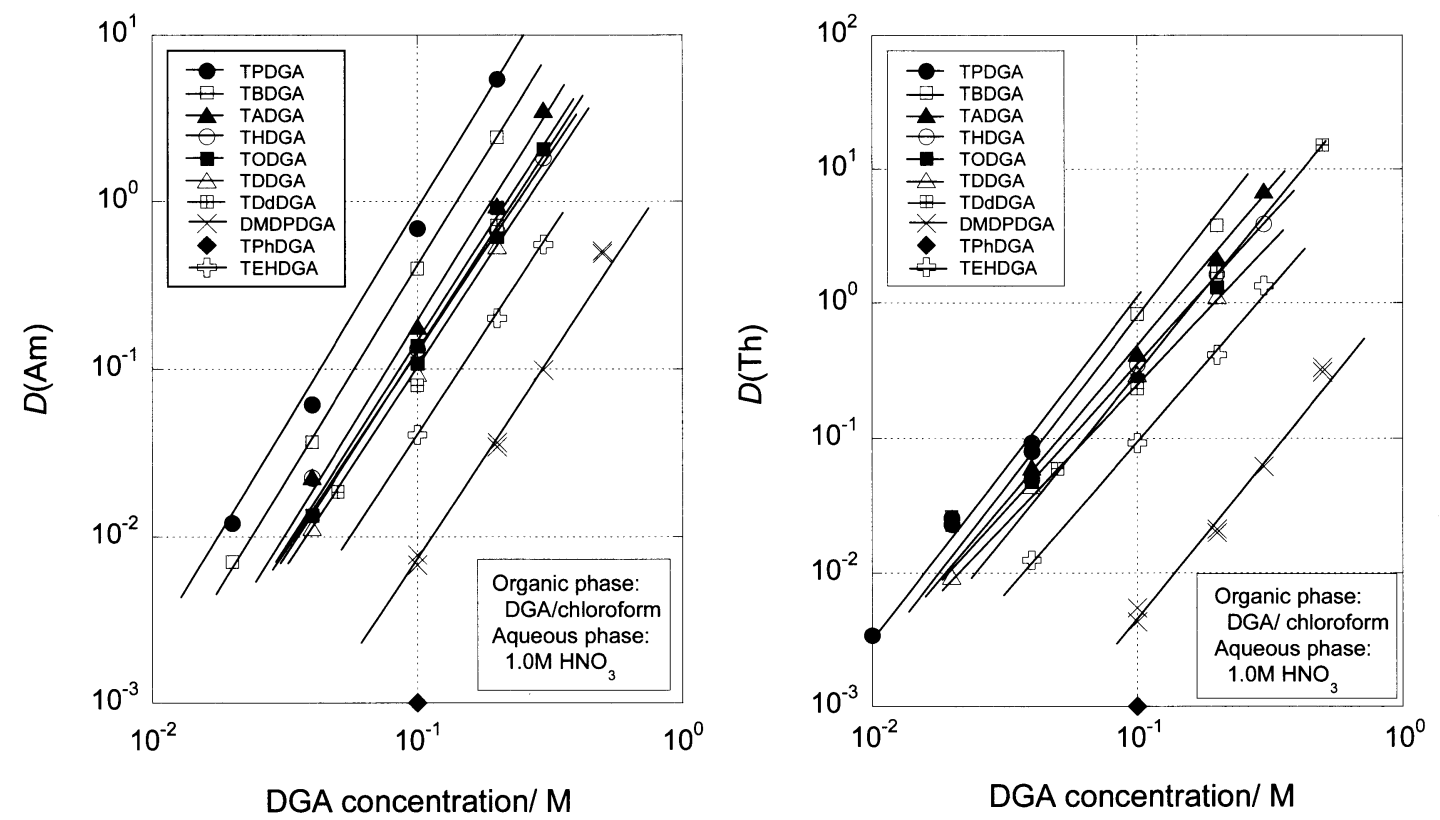

Fig. 5 Extraction behavior of Am(III) and Th(IV) by several DGA compounds Organic phase: DGA in chloroform; Aqueous phase: $1.0 \mathrm{M} \mathrm{HNO}_{3}$

\subsection{Effect of the functional groups attached to $\mathrm{N}$ atoms of DGA on extraction of An}

The extraction behavior of Am(III) and Th(IV) by several DGA compounds is illustrated in Fig. 5. Fig. 5 shows the relation between DGA concentration and $D(\mathrm{Am})$ or $D(\mathrm{Th})$. Here, the structures of novel DGA in Fig. 5 are as follows; tetradodecyl, dimethyldiphenyl, tetraphenyl, and tetra-2-ethylhexyl groups are attached to two $\mathrm{N}$ atoms of DGA, for the production of TDdDGA, DMDPDGA, TPhDGA, and TEHDGA, respectively. Here, the experiments were performed using chloroform for diluent of DGA, because TPDGA, DMDPDGA and TPhDGA have low solubility in nonpolar solvents. Fig. 5 shows the specific results described below”: (1) the $D$ values for Am(III) are similar to those for Th(IV), (2) DMDPDGA, TPhDGA, and TEHDGA have obviously the low $D$ values, compared with DGA having normal alkyl functional groups, and (3) the $D$ values decrease gradually with increase of carbon atom number in $n$ alkyl chains, with the slight difference in TH-, TO-, TD- and TDdDGA. Conventional extractants have the definitive difference of $D$ between An(III) and An(IV), and the usual order of $D$ for An of the different oxidation state is $\mathrm{An}(\mathrm{IV})>\mathrm{An}(\mathrm{VI})>\mathrm{An}(\mathrm{III})>\mathrm{An}(\mathrm{V})[10]$. Attachment of phenyl groups to $\mathrm{N}$ atoms of DGA reduces the stability of metal-

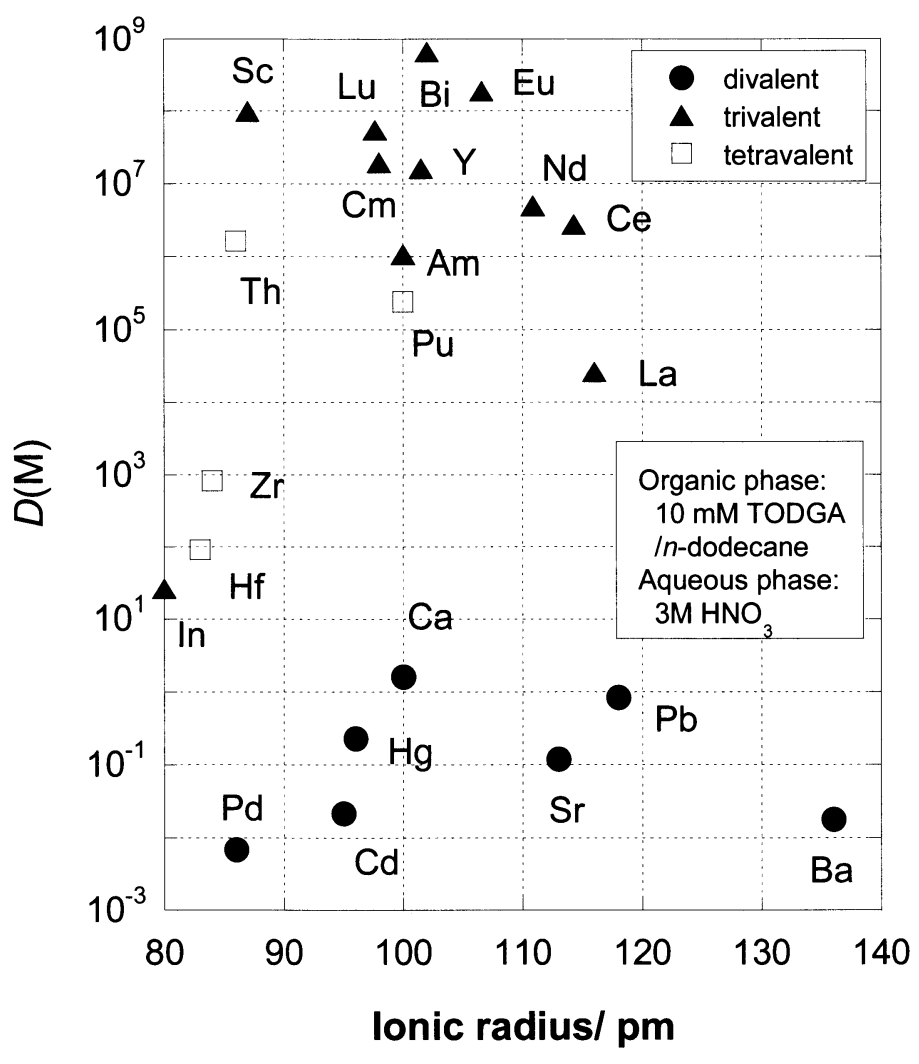

Fig. 6 Relationship between lonic radius of $M$ and $D$ values

Organic phase: $10 \mathrm{mM}$ TODGA/n-dodecane Aqueous phase: $3 \mathrm{M} \mathrm{HNO}_{3}$ 
complex, and this result is contradicted to the aryl substitution effect for the diphosphine dioxide[11]. Due to its steric hindrance, lengthening of alkyl chain may force the $D$ values to be changed and its variation is ambiguous at longer alkyl chains.

\subsection{Extraction of various metals by TODGA}

The extraction behavior of various metals by TODGA from nitric acid to $n$-dodecane was investigated. Fig. 6 shows the relation between ionic radius of metals and $D(\mathrm{M})$. Here some of the $D$ values in Fig. 6 were obtained from the extrapolation using the $\log D$ vs. $\log$ [TODGA] relations. The preliminary study suggested that the $D$ values of other metals, except the oxonium anions, were negligible small[12]. Although the $D$ values depend on their extraction reactions, it appears that the divalent, trivalent and tetravalent metals with ionic radius of $90-120 \mathrm{pm}$ have high $D$ values. This result suggests the size effect extraction by TODGA.

In conclusion, the diamide extractants having the different bridge between two amide groups in a molecule were investigated for the extraction of metals and An with the different valence states. The highest distribution ratio for An(III), (IV) and (VI) was demonstrated by DGA compound. The molar ratio of An:ligands measured by the slope analysis was mainly $1: 2$ and $1: 3$ for Am(III), $1: 1$ and $1: 2$ for Th(IV) and U(VI). TODGA and TDDGA with the carbon/oxygen $(\mathrm{C} / \mathrm{O})$ atom ratios of 12 and 14.7 exhibited high solubility in $n$-dodecane. DGA having the normal alkyl chain showed the high extractability, compared to those with the branched-

alkyl chain or the phenyl groups. TPDGA, TBDGA and TADGA had relatively high $D$ values with less solubility into non-polar diluents. Due to high extractability and the solubility in aliphatic solvents, TODGA, TDDGA and TDdDGA are recommended as extractant for recovery of An in nuclear fuel waste.

\section{Acknowledgments}

The authors acknowledge gratefully Professor Emeritus G.R. Choppin of Florida State University and Drs. Y. Morita, S Suzuki, and Y. Sugo of Japan Atomic Energy Agency for the useful discussion and the experimental supports in the laboratory.

\section{References}

1) C. Madic, P. Blanc, N. Condamines, P. Baron, L. Berthon, C. Nicol, C. Pozo, M. Lecomte, M. Philippe, M. Masson, C. Hequet, M.J. Hudson, CEA-CONF-12297, (1994).

2) X.F. Tan, Y.S. Wang, T.Z. Tan, G.F. Zhou, B.R. Bao, J. Radioanal. Nucl. Chem., Articles 242, 123-126(1999).

3) C. Shen, B. Bao, J. Zhu, Y. Wang, Z. Cao, J. Radioanal. Nucl. Chem., Articles, 212, 187-196 (1996).

4) M.C. Charbonne, C. Musikas, Solv. Extr. Ion Exch., 6, 461-478 (1998).

5) L. Spjuth, J.O. Liljenzin, M. Skalberg, M.J. Hudson, G.Y.S. Chan, M.G.B. Drew, M. Feaviour, P.B. Iveson, C. Madic, Radiochim. Acta, 78, 39-46 (1997).

6) T.H. Siddall III, M.L. Good, J. Inorg. Nucl. Chem., 29, 149-158 (1967).

7) Y. Sasaki, G.R. Choppin, Anal. Sci., 12, 225-230 (1996).

8.) Y. Sasaki, G.R. Choppin, Radiochim. Acta, 80, 85-88 (1998).

9) G. M. Gasparini, G. Grossi, Solv. Extr. Ion Exch., 4, 1233-1271 (1986).

10) G.R. Choppin, "Separation processes for actinide elements," Sep. Sci. Tech., 19, 911-925 (1984-1985).

11) A.M. Rozen, J. Radioanal. Nucl. Chem., Articles, 143, 337-355 (1990).

12) Z.-X. Zhu, Y. Sasaki, H. Suzuki, S. Suzuki, T. Kimura, Anal. Chim. Acta, 527, 163-168 (2004). 